\title{
GLOBAL WELL-POSEDNESS AND SCATTERING FOR DEFOCUSING ENERGY-CRITICAL NLS IN THE EXTERIOR OF BALLS WITH RADIAL DATA
}

\author{
Dong Li, Hart Smith AND XiaOyi Zhang
}

\begin{abstract}
We consider the defocusing energy-critical nonlinear Schrödinger (NLS) in the exterior of the unit ball in three dimensions. For the initial value problem with Dirichlet boundary condition, we prove global well-posedness and scattering with large radial initial data in the Sobolev space $\dot{H}_{0}^{1}$. We also point out that the same strategy can be used to treat the energy-supercritical NLS in the exterior of balls with Dirichlet boundary condition and radial $\dot{H}_{0}^{1}$ initial data.
\end{abstract}

\section{Introduction}

Let $\Omega=\mathbb{R}^{3} \backslash \bar{B}(0,1)$ be the exterior of the unit ball. We consider the defocusing energy critical NLS in $\Omega$ with Dirichlet boundary condition:

$$
\left\{\begin{array}{l}
i \partial_{t} u+\Delta u=|u|^{4} u \equiv F(u),(t, x) \in \mathbb{R} \times \Omega, \\
\left.u(t, x)\right|_{\mathbb{R} \times \partial \Omega}=0, \\
u(0, x)=u_{0}(x) .
\end{array}\right.
$$

Our main purpose is to prove the global solvability and scattering for the solution to (1.1) under the assumption that $u_{0} \in \dot{H}_{0}^{1}(\Omega)$ (see Section 2.4 for the definition), and that $u_{0}$ is spherically symmetric.

In the whole space case $\mathbb{R}^{n}$ with $n \geq 3$, the Cauchy problem for the energy critical nonlinear Schrödinger (NLS) has been successfully attacked in both defocusing and focusing cases $[6,8,13-15,19,21]$. On the other hand, the understanding of the critical nonlinear problem of NLS posed on exterior domains is still unsatisfactory. The difficulty comes from several aspects. First of all, concerning linear estimates, the dispersive estimates and Strichartz estimates are not always available and often more limited than the whole space case. Secondly, the nonlinear problem no longer has translation invariance or scale invariance, and many of the technical tools built on frequency analysis are not immediately applicable in the obstacle case.

The Strichartz estimates on exterior domains or more general Riemannian manifolds are usually obtained by using local smoothing estimates $[4,9,16,20]$ combined with semi-classical parametrix constructions. For the domain exterior to a non-trapping obstacle in $\mathbb{R}^{n}$, Blair et al. [4] obtained a range of scale-invariant Strichartz estimates; in particular the endpoint $L_{t}^{4} L_{x}^{\infty}$ estimate in dimension $n=3$, by using a microlocal parametrix previously used for the wave equation in $[5,18]$. For the exterior domain to a strictly convex obstacle, i.e., $\Omega=\mathbb{R}^{n} \backslash \mathcal{K}$, where $\mathcal{K}$ is strictly convex, Ivanovici [11] obtained the full range of Strichartz estimates excepting endpoints, by

Received by the editors May 17, 2011. 
using the Melrose-Taylor parametrix construction. For Strichartz estimates with loss of derivatives; see $[1,2,10]$.

For the energy-critical nonlinear wave equation in three-dimensional smooth bounded domains with Dirichlet boundary condition, Burq et al. [3] established global well-posedness for $H_{0}^{1}$ solutions. Previously, Smith and Sogge [17] proved global wellposedness for the corresponding problem on the exterior domain to a strictly convex obstacle.

In this paper, we shall prove the global well-posedness and scattering of energycritical NLS outside the unit ball in $\mathbb{R}^{3}$ under the radial assumption. Our result is the following.

Theorem 1.1. Let $\Omega=\mathbb{R}^{3} \backslash \bar{B}(0,1)$. Let $u_{0} \in \dot{H}_{0}^{1}(\Omega)$ be spherically symmetric. Then there exists a unique solution $u \in C_{t}^{0} \dot{H}_{0}^{1}(\mathbb{R} \times \Omega)$ to (1.1), and

$$
\|u\|_{L_{t, x}^{10}(\mathbb{R} \times \Omega)} \leq C\left(\left\|u_{0}\right\|_{\dot{H}_{0}^{1}(\Omega)}\right) .
$$

Moreover, there exists unique $v_{ \pm} \in \dot{H}_{0}^{1}(\Omega)$ such that

$$
\lim _{t \rightarrow \pm \infty}\left\|u(t)-e^{i t \Delta_{D}} v_{ \pm}\right\|_{\dot{H}_{0}^{1}(\Omega)}=0 .
$$

Here, $\Delta_{D}$ is the Dirichlet Laplacian and $e^{i t \Delta_{D}}$ is the free propagator.

Remark 1.1. The assumption $u_{0} \in \dot{H}_{0}^{1}(\Omega)$ is very natural here due to the energy critical nature of the problem. On the other hand, if we assume $u_{0} \in H_{0}^{1}(\Omega)$, then the proof can be trivialized; see Section 5 for details.

The proof of Theorem 1.1 follows roughly the strategy in the paper by Bourgain [6] for dimension $n=3,4$ and Tao [19] for all dimensions, which dealt with defocusing energy critical NLS in the whole-space case with radial data. However, since many technical tools are missing in this setting, we devote a large portion of the work to establishing the technical tools in analogy with the whole space case. A crucial fact exploited in this paper is that under the radial assumption, the eigenfunctions of the Dirichlet Laplacian in the domain exterior to a ball can be explicitly computed. We then use this explicit knowledge to establish the following basic estimates.

- The fundamental solution is written explicitly through spectral representation of Dirichlet Laplacian. The $L^{1}-L^{\infty}$ dispersive estimate then follows from the explicit representation of the linear solution. As a consequence, we prove the full range Strichartz estimates with no loss of derivatives.

- The Littlewood-Paley operators are defined through functional calculus. Bernstein type estimates for the Littlewood-Paley operators are also shown to hold true.

- Sobolev spaces on the exterior domain $\dot{H}_{0}^{1, p}(\Omega)$ and $\dot{H}_{D}^{1, p}(\Omega)$ for $1<p<3$ (see Section 2.4) are proved to be equivalent. Therefore the product rule and chain rule for the Dirichlet Laplacian is still applicable as in the whole space case.

With these technical tools in hand, we reduce Theorem 1.1 to establishing the a priori bound of the $L_{t, x}^{10}$-norm of the solution. Then we follow the spirit of the argument in $[6,19]$. Whilst a handful of estimates still hold true as in the whole space 
case, the most problematic part comes from proving the mass localization. In the whole space case, the key fact used in the proof is that the Littlewood-Paley operator is defined through convolution with a normalized Schwartz function. This property no longer holds in our setting since we do not have translation-invariance. Alternatively, we shall prove the localization though a careful analysis on the interaction between spatial and frequency cutoffs. ${ }^{1}$

In Section 2, we introduce basic notations and some useful estimates. The proof of Theorem 1.1 is contained in Section 3. We shall only focus on the parts, which are different from the whole space case: the mass localization and the Morawetz inequality. The combinatorics argument, which eventually gives the upper bound of the space-time norm of the solution, will only be sketched. One can refer to [6] or [19] for more details. In Sections 4 and 5, we give remarks on the energy supercritical problem and the case with inhomogeneous Sobolev data.

\section{Basic estimates}

2.1. Some notation. We write $X \lesssim Y$ or $Y \gtrsim X$ to indicate $X \leq C Y$ for some non-essential constant $C>0$. We use $\mathcal{O}(Y)$ to denote any quantity $X$ such that $|X| \lesssim Y$. We use the notation $X \sim Y$ whenever $X \lesssim Y \lesssim X$. The fact that these constants depend upon the dimension $d$ will be suppressed. If $C$ depends upon some additional parameters, we will indicate this with subscripts; for example, $X \lesssim_{u} Y$ denotes the assertion that $X \leq C_{u} Y$ for some $C_{u}$ depending on $u$. Sometimes when the context is clear, we will suppress the dependence on $u$ and write $X \lesssim{ }_{u} Y$ as $X \lesssim Y$. We will write $C=C\left(Y_{1}, \ldots, Y_{n}\right)$ to stress that the constant $C$ depends on quantities $Y_{1}, \ldots, Y_{n}$.

Let $I \subset \mathbb{R}$ be a time interval. We write $L_{t}^{q} L_{x}^{r}(I \times \Omega)$ to denote the Banach space with norm

$$
\|u\|_{L_{t}^{q} L_{x}^{r}(I \times \Omega)}:=\left(\int_{I}\left(\int_{\Omega}|u(t, x)|^{r} d x\right)^{q / r} d t\right)^{1 / q},
$$

with the usual modifications when $q$ or $r$ are equal to infinity. When $q=r$ we abbreviate $L_{t}^{q} L_{x}^{q}$ as $L_{t, x}^{q}$. We shall write $u \in L_{t, l o c}^{q} L_{x}^{r}(I \times \Omega)$ if $u \in L_{t}^{q} L_{x}^{r}(J \times \Omega)$ for any compact $J \subset I$.

For any positive number $1 \leq a \leq \infty$, we let $a^{\prime}=a /(a-1)$ denote the conjugate of $a$, so that $1 / a+1 / a^{\prime}=1$.

We use $\mathcal{S}(\mathbb{R})$ to denote the space of Schwartz functions, and $\mathcal{S}^{\prime}(\mathbb{R})$ the space of tempered distributions, on the real line.

2.2. Fundamental solution and Strichartz estimates. The spectral resolution for radial functions on the exterior domain $r \geq 1$ in $\mathbb{R}^{3}$ is expressed using the radial eigenfunctions

$$
\Delta \phi_{\lambda}+\lambda^{2} \phi_{\lambda}=0
$$

for $\lambda>0$ which satisfy the Sommerfeld radiation condition, namely

$$
\phi_{\lambda}(r)=\frac{\sin \lambda(r-1)}{r}, \quad r \geq 1
$$

\footnotetext{
${ }^{1}$ In the language of [19], we actually showed that the length of unexceptional intervals must have a uniform lower bound. This is quite different from the whole-space case.
} 
For tempered distributions $f \in \mathcal{S}^{\prime}(\mathbb{R})$, we set

$$
\mathcal{F} f(\lambda)=\frac{1}{\sqrt{\pi}} \int \frac{\sin \lambda(s-1)}{s} f(s) s^{2} d s
$$

which can be expressed in terms of the Fourier transform of $s f(s)$ to identify $\mathcal{F} f$ as an odd element of $\mathcal{S}^{\prime}(\mathbb{R})$. If $f \in \mathcal{S}(\mathbb{R})$, then $\mathcal{F} f$ is a Schwartz function of $\lambda$.

We observe the following resolution of identity:

$$
\begin{aligned}
\frac{1}{\pi} \int_{-\infty}^{\infty} \phi_{\lambda}(r) \phi_{\lambda}(s) d \lambda & =\frac{1}{2 \pi r s} \int_{-\infty}^{\infty} \cos \lambda(r-s)-\cos \lambda(r+s-2) d \lambda \\
& =\frac{\delta(r-s)+\delta(r+s-2)}{r s} \\
& =\frac{\delta(r-s)}{s^{2}}, \quad \text { for } r, s>1,
\end{aligned}
$$

from which it follows by a limiting procedure that $\mathcal{F}^{*} \mathcal{F} f=f$ for $f \in \mathcal{S}$ supported in $[1, \infty)$, where $\mathcal{F}^{*}$ is the formal adjoint, defined on tempered distributions $g$ as the restriction to $r \neq 0$ of

$$
\mathcal{F}^{*} g=\frac{1}{\sqrt{\pi}} \int_{-\infty}^{\infty} \frac{\sin \lambda(r-1)}{r} g(\lambda) d \lambda .
$$

Consequently, $f \rightarrow \mathcal{F}_{0} f=\left.\sqrt{2} \mathcal{F} f\right|_{\lambda>0}$ induces an isometric map

$$
\mathcal{F}_{0}: L^{2}\left([1, \infty), s^{2} d s\right) \rightarrow L^{2}([0, \infty), d \lambda) .
$$

One can similarly verify that if $g$ is an odd element of $\mathcal{S}(\mathbb{R})$, then

$$
\frac{2}{\pi} \int_{1}^{\infty} \int_{0}^{\infty} \frac{\sin \lambda(r-1)}{r} \frac{\sin \mu(r-1)}{r} g(\mu) d \mu r^{2} d r=g(\lambda),
$$

hence $\mathcal{F}_{0}$ in $(2.1)$ is onto, and thus an isomorphism of Hilbert spaces.

We will also use the radial inhomogeneous Sobolev space $\dot{H}_{0}^{1}(\Omega)$, defined as the closure of $C_{c}^{\infty}([1, \infty))$ in the norm

$$
\|f\|_{\dot{H}_{0}^{1}}=\left\|f^{\prime}(r)\right\|_{L^{2}\left(r^{2} d r\right)} .
$$

By Sobolev embedding on $\mathbb{R}^{3}$, for compactly supported $f$, we have

$$
\|f\|_{L^{6}\left(r^{2} d r\right)} \leq\left\|f^{\prime}\right\|_{L^{2}\left(r^{2} d r\right)} .
$$

Conversely, if $f \in L^{6}\left(r^{2} d r\right)$ and $f^{\prime} \in L^{2}\left(r^{2} d r\right)$, then $\chi\left(N^{-1} r\right) f$ converges to $f$ in the $\dot{H}_{0}^{1}$ norm, hence we may identify $\dot{H}_{0}^{1}(\Omega)$ as absolutely continuous functions on $[1, \infty)$ for which

$$
\left\|f^{\prime}\right\|_{L^{2}\left(r^{2} d r\right)}+\|f\|_{L^{6}\left(r^{2} d r\right)}<\infty, \quad f(1)=0
$$

If $f \in C_{c}^{\infty}(\Omega)$ is radial, then $\mathcal{F}_{0}(\Delta f)(\lambda)=-\lambda^{2} \mathcal{F}_{0} f(\lambda)$. On the other hand,

$$
\int_{\Omega} \bar{f} \Delta f d x=\int_{\Omega}|\nabla f|^{2} d x=\int_{\Omega}\left|f^{\prime}\right|^{2} d x
$$

hence by $(2.1), \mathcal{F}_{0}$ induces an isometric map

$$
\mathcal{F}_{0}: \dot{H}_{0}^{1}(\Omega) \rightarrow L^{2}([0, \infty), \lambda d \lambda) .
$$

The image contains odd Schwartz functions by (2.2), hence is an isomorphism of Hilbert spaces. 
Finally, we observe that

$$
|f(r)| \leq r^{-\frac{1}{2}}\left(\int_{r}^{\infty}\left|f^{\prime}(s)\right|^{2} s^{2} d s\right)^{\frac{1}{2}}, \quad \text { hence } \quad\left\||x|^{\frac{1}{2}} f\right\|_{L^{\infty}} \leq\|f\|_{\dot{H}_{0}^{1}} .
$$

It follows as an easy consequence that radial $\dot{H}_{0}^{1}$ is an algebra under multiplication of functions.

For $f \in L^{2}+\dot{H}_{0}^{1}$ (see Section 2.3 below), we can express the Schrödinger propagator $e^{i t \Delta_{D}}$ as

$$
\left(e^{i t \Delta_{D}} f\right)(r, t)=\mathcal{F}_{0}^{*}\left(e^{-i \lambda^{2} t} \mathcal{F}_{0} f\right)(r) .
$$

The corresponding kernel is, where $t= \pm|t|$,

$$
\begin{aligned}
K(t, r, s) & =\frac{2}{\pi} \int_{0}^{\infty} \frac{\sin \lambda(r-1)}{r} \cdot \frac{\sin \lambda(s-1)}{s} \cdot e^{-i \lambda^{2} t} d \lambda \\
& =\frac{1}{\pi r s} \int_{-\infty}^{\infty}\left(e^{i \lambda(r-s)}-e^{i \lambda(r+s-2)}\right) e^{-i \lambda^{2} t} d \lambda \\
& =\frac{\pi^{\frac{1}{2}} e^{ \pm i \pi / 4}}{|t|^{\frac{1}{2}} r s}\left(e^{i(r-s)^{2} / 4 t}-e^{i(r+s-2)^{2} / 4 t}\right) \\
& =\frac{\pi^{\frac{1}{2}} e^{ \pm i \pi / 4}}{|t|^{\frac{1}{2}} r s}\left(1-e^{i(r-1)(s-1) / t}\right) .
\end{aligned}
$$

It follows that $|K(t, r, s)| \leq C|t|^{-3 / 2}$ for $r, s \geq 1$. By a density argument, we thus have the important

Lemma 2.1 (Dispersive estimate). For $t \neq 0$ and radial $f \in L^{2}+\dot{H}_{0}^{1}$, we have

$$
\left\|e^{i t \Delta_{D}} f\right\|_{L^{\infty}(\Omega)} \lesssim \frac{1}{|t|^{\frac{3}{2}}}\|f\|_{L^{1}(\Omega)} .
$$

Strichartz estimates for radial data follow directly from this dispersive estimate. See [12] for instance. Therefore, we have the following lemma whose proof will be omitted.

Lemma 2.2. Let $I$ be a time interval containing 0 . Let $u(t, x)$ satisfy

$$
u(t, \cdot)=e^{i t \Delta_{D}} u_{0}-i \int_{0}^{t} e^{i(t-s) \Delta_{D}} f(s, \cdot) d s, \quad \forall t \in I,
$$

where $u_{0} \in L^{2}+\dot{H}_{0}^{1}, f \in L_{t}^{1}\left(L^{2}+\dot{H}_{0}^{1}\right)$, with both radial.

Let $\left(q_{i}, r_{i}\right), i=1,2$ be admissible pairs, such that $2 \leq q_{i} \leq \infty, \frac{2}{q_{i}}+\frac{3}{r_{i}}=\frac{3}{2}$. Then

$$
\|u\|_{L_{t}^{q_{1}} L_{x}^{r_{1}(I \times \Omega)}} \lesssim\left\|u_{0}\right\|_{L^{2}(\Omega)}+\|f\|_{L_{t}^{q_{2}^{\prime}} L_{x}^{r_{2}^{\prime}}(I \times \Omega)} .
$$

Here $\left(q_{2}^{\prime}, r_{2}^{\prime}\right)$ are the conjugate exponents of $\left(q_{2}, r_{2}\right)$.

2.3. Littlewood-Paley operators and Bernstein inequalities. Given a bounded function $m(\lambda)$, which for convenience we assume to be defined on all of $\mathbb{R}$ and even in $\lambda$, we define

$$
m\left(\sqrt{-\Delta_{D}}\right) f=\mathcal{F}_{0}^{*}\left(m(\cdot) \mathcal{F}_{0} f\right) .
$$


This defines a functional calculus on $L^{2}+\dot{H}_{0}^{1}$. In this section, we will take $m$ to be an even function in $C_{c}^{\infty}(\mathbb{R})$, in which case

$$
m\left(\sqrt{-\Delta_{D}}\right) f(r)=\int_{1}^{\infty} K_{m}(r, s) f(s) s^{2} d s,
$$

with

$$
K_{m}(r, s)=\frac{1}{\pi} \cdot \frac{\widehat{m}(r-s)-\widehat{m}(r+s-2)}{r s} .
$$

In particular, we can define, for $N>0$, Littlewood-Paley projectors $P_{N}$ by taking $m=\psi\left(N^{-1} \lambda\right)$, for suitable $\psi$ compactly supported away from 0 . We similarly define $P_{\leq N}$ using $m=\phi\left(N^{-1} \lambda\right)$, where $\phi \in C_{c}^{\infty}(\mathbb{R})$ equals 1 on a neighborhood of 0 . We also set $P_{\geq N}=1-P_{\leq N}$.

Remark 2.1. An added complication for this work, relative to the whole space case, is that that spectral supports are not additive under function multiplication, and thus we cannot exploit standard paraproduct decomposition results.

As in the whole space case, we have the following

Proposition 2.1 (Bernstein inequality). Let $1 \leq p \leq q \leq \infty$, and suppose $\sigma \in \mathbb{R}$. Then for any $N>0$

$$
\begin{aligned}
\left\|P_{\leq N} f\right\|_{L^{q}(\Omega)} & \lesssim N^{3\left(\frac{1}{p}-\frac{1}{q}\right)}\|f\|_{L^{p}(\Omega)}, \\
\left\|\left(-\Delta_{D}\right)^{\frac{\sigma}{2}} P_{N} f\right\|_{L^{p}(\Omega)} & \approx N^{\sigma}\left\|P_{N} f\right\|_{L^{p}(\Omega)} .
\end{aligned}
$$

Proof. We first prove (2.4). We write

$$
\left(P_{\leq N} f\right)(r)=\int_{1}^{\infty} K_{N}(r, s) f(s) s^{2} d s
$$

where

$$
K_{N}(r, s)=\frac{N}{\pi} \cdot \frac{\widehat{\phi}(N(r-s))-\widehat{\phi}(N(r+s-2))}{r s},
$$

and we observe that $\widehat{\phi}$ is an even Schwartz function. Since $K$ is symmetric, it suffices by the Schur test and interpolation to show that

$$
\begin{aligned}
\sup _{r}\left\|K_{N}(r, s)\right\|_{L^{1}\left(s^{2} d s\right)} & \leq C, \\
\sup _{r, s}\left|K_{N}(r, s)\right| & \leq C N^{3} .
\end{aligned}
$$

We pose $r=1+N^{-1} x, s=1+N^{-1} y$, where $x, y>0$. Since $\frac{s}{r} \leq \frac{y}{x}+1$, then (2.6) is implied by the bound

$$
\begin{gathered}
\int_{0}^{\infty}|\widehat{\phi}(y+x)-\widehat{\phi}(y-x)| d y \leq C, \\
\int_{0}^{\infty}|\widehat{\phi}(y+x)-\widehat{\phi}(y-x)| y d y \leq C x .
\end{gathered}
$$

(2.8) is easy to see with the bound $2\|\hat{\phi}\|_{1}$. For $y>2 x$, we can bound $\mid \widehat{\phi}(y+x)-\widehat{\phi}(y-$ $x) \mid \lesssim x(1+y)^{-4}$, which establishes $(2.9)$ for the integral over $y>2 x$. The remaining piece can be bounded directly by $4 x\|\hat{\phi}\|_{1}$. 
For (2.7), we use the evenness of $\widehat{\phi}$ to write $\widehat{\phi}(s)=g\left(s^{2}\right)$, where $g$ is Schwartz, to bound

$$
|K(r, s)| \leq 4 N^{3}\left|\frac{g\left((x+y)^{2}\right)-g\left((x-y)^{2}\right)}{(x+y)^{2}-(x-y)^{2}}\right| .
$$

The inequality (2.7) follows, where we bound $\left|g^{\prime}\right| \leq C / 4$.

Relation (2.5) follows similarly, by writing $\lambda^{\sigma} \psi\left(N^{-1} \lambda\right)=N^{\sigma} \tilde{\psi}\left(N^{-1} \lambda\right) \psi\left(N^{-1} \lambda\right)$ where $\tilde{\psi} \in C_{c}^{\infty}(\mathbb{R})$, and applying (2.6) for the kernel associated to $\tilde{\psi}\left(N^{-1} \lambda\right)$.

2.4. $\boldsymbol{L}^{\boldsymbol{p}}$ based Sobolev spaces. We will also have need to work with the inhomogeneous Sobolev norm for radial functions, $\|f\|_{\dot{H}_{0}^{1, p}(\Omega)}=\|\nabla f\|_{L^{p}(\Omega)}=\left\|f^{\prime}\right\|_{L^{p}\left(s^{2} d s\right)}$. The difficulty with using this norm is that $\nabla$ does not commute with $e^{i t \Delta_{D}}$. This problem is solved by proving an equivalence

$$
\|f\|_{\dot{H}_{0}^{1, p}(\Omega)} \approx\left\|\left(-\Delta_{D}\right)^{\frac{1}{2}} f\right\|_{L^{p}(\Omega)} .
$$

If $p>3$ this cannot hold for all $f \in \dot{H}_{0}^{1, p}$, as seen by taking $f=\frac{\sin \lambda(r-1)}{\lambda r}$, where the right side tends to 0 as $\lambda \rightarrow 0$, but the left side remains bounded below. However, we shall only need to apply this equivalence for $1<p<3$ and for $f \in \dot{H}_{0}^{1,2}=\dot{H}_{0}^{1}$, for which it does hold. Note that in this case, both $|\nabla f|=\left|f^{\prime}\right|$ and $\left(-\Delta_{D}\right)^{\frac{1}{2}} f$ belong to $L^{2}(\Omega)$, so both sides of the equivalence are well defined.

Proposition 2.2. Let $1<p<3$. Then there exists a constant $0<C_{p}<\infty$, such that for any radial function $f \in \dot{H}_{0}^{1}$, we have

$$
C_{p}^{-1}\|\nabla f\|_{L^{p}(\Omega)} \leq\left\|\left(-\Delta_{D}\right)^{\frac{1}{2}} f\right\|_{L^{p}(\Omega)} \leq C_{p}\|\nabla f\|_{L^{p}(\Omega)} .
$$

Proof. We first establish that

$$
\left\|\left(-\Delta_{D}\right)^{\frac{1}{2}} f\right\|_{L^{p}(\Omega)} \lesssim\left\|f^{\prime}\right\|_{L^{p}(\Omega)}
$$

We will establish this under the assumption that $f(r) \in C_{c}^{\infty}([1, \infty))$. To establish it for general $f$, we take a sequence $f_{j} \in C_{c}^{\infty}([1, \infty))$ with $\left\|f_{j}^{\prime}-f^{\prime}\right\|_{L^{q}(\Omega)} \leq 2^{-j}$ for both $q=2$ and $q=p$. It follows that $\left\|\left(-\Delta_{D}\right)^{\frac{1}{2}}\left(f_{j}-f\right)\right\|_{L^{2}(\Omega)} \lesssim 2^{-j}$, hence

$$
\left(-\Delta_{D}\right)^{\frac{1}{2}} f_{j} \rightarrow\left(-\Delta_{D}\right)^{\frac{1}{2}} f \text { pointwise a.e. }
$$

The result for general $f \in \dot{H}_{0}^{1}$ then follows by Fatou's lemma.

For $f \in C_{c}^{\infty}$ we write

$$
\begin{aligned}
\left(\left(-\Delta_{D}\right)^{\frac{1}{2}} f\right)(r) & =\frac{2}{\pi} \int_{0}^{\infty} \int_{1}^{\infty} \frac{\sin \lambda(r-1)}{r} \frac{\sin \lambda(s-1)}{s} \lambda f(s) s^{2} d s d \lambda \\
& =\frac{2}{\pi} \int_{0}^{\infty} \int_{1}^{\infty} \frac{\sin \lambda(r-1)}{r}\left(s \cos \lambda(s-1)-\frac{1}{\lambda} \sin \lambda(s-1)\right) f^{\prime}(s) d s d \lambda .
\end{aligned}
$$

By considering the limit of the truncated integrals over $\lambda$, we obtain

$$
\left(\left(-\Delta_{D}\right)^{\frac{1}{2}} f\right)(r)=-\frac{2}{\pi} \int_{1}^{\infty} K_{1}(r, s) f^{\prime}(s) s^{2} d s,
$$

where

$$
K_{1}(r, s)=\frac{1}{r s}\left(\frac{1}{r+s-2}+\frac{1}{r-s}\right)+\frac{1}{r s^{2}} \log \left|\frac{r-s}{r+s-2}\right|
$$


and the first term is interpreted as a principal value integral. We note that this kernel is only applied to functions $f^{\prime}(s)$ of integral 0 , hence we can add a function $k(r) s^{-2}$ to $K_{1}(r, s)$ without changing the result. This will indeed be necessary for small $p$.

A similar computation, using the whole-space spectral decomposition for radial functions

$$
\left((-\Delta)^{\frac{1}{2}} f\right)(r)=\frac{2}{\pi} \int_{0}^{\infty} \int_{0}^{\infty} \frac{\sin \lambda r}{r} \frac{\sin \lambda s}{s} \lambda f(s) s^{2} d s d \lambda,
$$

expresses $(-\Delta)^{\frac{1}{2}} f(r)=-\frac{2}{\pi} \int_{0}^{\infty} K_{0}(r, s) f^{\prime}(s) s^{2} d s$, with the kernel

$$
K_{0}(r, s)=\frac{1}{r s}\left(\frac{1}{r+s}+\frac{1}{r-s}\right)+\frac{1}{r s^{2}} \log \left|\frac{r-s}{r+s}\right| .
$$

This kernel is bounded on $L^{p}\left(s^{2} d s\right), 1<p<\infty$, since ${ }^{2}$ it represents the operator

$$
g \rightarrow \sum_{j=1}^{3} R_{j}\left(\frac{x_{j}}{|x|} g\right)
$$

where $R_{j}$ is the Riesz transform $\partial_{x_{j}}(-\Delta)^{-\frac{1}{2}}$ on $\mathbb{R}^{3}$.

We are thus reduced to proving $L^{p}\left([1, \infty), s^{2} d s\right)$ boundedness of the kernel $K=$ $K_{1}-K_{0}$, that is

$$
K(r, s)=\frac{1}{r s}\left(\frac{1}{r+s-2}-\frac{1}{r+s}\right)+\frac{1}{r s^{2}} \log \left(\frac{r+s}{r+s-2}\right),
$$

with the freedom to add $k(r) s^{-2}$ to $K(r, s)$. Note that both terms on the right of (2.11) are non-negative, hence can be considered separately. The $L^{p}$ boundedness of the first term is based on the bound, for $1 \leq p<\infty$,

$$
\left\|\frac{2}{r s(r+s-2)(r+s)}\right\|_{L^{p^{\prime}\left(s^{2} d s\right)}} \lesssim\left[r^{2}(r-1)\right]^{-p}, \quad r>1 .
$$

This shows that the corresponding operator is weak-type $(p, p)$ for $1 \leq p<\infty$, hence strong-type $(p, p)$ for $1<p<\infty$.

For the second term on the right of (2.11), we note that if $1 \leq r \leq 2$ then for each $1<p^{\prime}<\infty$ the $L^{p^{\prime}}\left(s^{2} d s\right)$ norm is bounded uniformly in $r$.

For $r>2$, we write the second term as

$$
-\frac{1}{r s^{2}} \log \left(1-\frac{2}{r+s}\right)=\frac{2}{r s^{2}(r+s)}+\mathcal{O}\left(\frac{1}{r s^{2}(r+s)^{2}}\right) .
$$

The second term on the right-hand side of (2.12) is bounded by the first term in $K(r, s)$ considered above. If $1<p^{\prime}<3$, then we dominate the $L^{p^{\prime}}$ norm of the first term on the right-hand side of $(2.12)$ by

$$
\left\|\frac{1}{s(r+s)^{2}}\right\|_{L^{p^{\prime}\left((0, \infty), s^{2} d s\right)}}=C r^{-\frac{3}{p}} .
$$

This implies strong-type $(p, p)$ bounds for the second term in $(2.11)$ if $\frac{3}{2}<p<\infty$.

\footnotetext{
${ }^{2}$ This can be verified directly by elementary computation. Alternatively, the two operators must agree on $g$ of integral 0 , determining $K_{0}(r, s)$ up to $k(r) s^{-2}$. Since $K_{0}$ decrease like $s^{-3}$ as $s \rightarrow \infty$, then necessarily $k(r)=0$.
} 
To obtain $(p, p)$ bounds for remaining $p$, we consider $1<p<3$, and subtract the kernel $2 / r^{2} s^{2}$. Since this kernel is bounded in $L^{p^{\prime}}\left(s^{2} d s\right)$ for $p^{\prime}>\frac{3}{2}$, it does not affect the above consideration for $1<r<2$. For $r>2$, we are reduced to considering

$$
\frac{2}{r^{2} s^{2}}-\frac{2}{r s^{2}(r+s)}=\frac{2}{r^{2} s(r+s)} \leq \frac{4}{r(r+s)^{2}} \text {. }
$$

We conclude by observing that, for $p^{\prime}>\frac{3}{2}$,

$$
\left\|\frac{1}{r(r+s)^{2}}\right\|_{L^{p^{\prime}\left((0, \infty), s^{2} d s\right)}}=C r^{-\frac{3}{p}}
$$

which yields the strong-type $(p, p)$ bounds for $1<p<3$. Note that we have in fact established (2.10) for all $1<p<\infty$.

To show the reverse implication, for $1<p<3$,

$$
\|\nabla f\|_{L^{p}(\Omega)} \lesssim\left\|\left(-\Delta_{D}\right)^{\frac{1}{2}} f\right\|_{L^{p}(\Omega)}, \quad \text { if } f \in \dot{H}_{0}^{1},
$$

we consider $f_{N}=P_{\leq N} f$. By $(2.4)$,

$$
\left\|\left(-\Delta_{D}\right)^{\frac{1}{2}} f_{N}\right\|_{L^{p}(\Omega)} \lesssim\left\|\left(-\Delta_{D}\right)^{\frac{1}{2}} f\right\|_{L^{p}(\Omega)} .
$$

Since $\left\|f_{N}^{\prime}-f^{\prime}\right\|_{L^{2}(\Omega)} \rightarrow 0$, for some subsequence $f_{N_{j}}^{\prime}(r) \rightarrow f^{\prime}(r)$ pointwise a.e. By Fatou's lemma, it thus suffices to prove (2.13) for $f \in \dot{H}_{0}^{1}$ with compact spectral support in $[0, \infty)$. Such functions are smooth, as is $\left(-\Delta_{D}\right)^{\frac{1}{2}} f$.

For such $f$, we can write

$$
\begin{aligned}
f^{\prime}(r) & =\frac{2}{\pi} \int_{0}^{\infty} \frac{\partial}{\partial r}\left(\frac{\sin \lambda(r-1)}{\lambda r}\right) \mathcal{F}_{0}\left(\left(-\Delta_{D}\right)^{\frac{1}{2}} f\right)(\lambda) d \lambda \\
& =\frac{2}{\pi} \int_{0}^{\infty}\left(\frac{\cos \lambda(r-1)}{r}-\frac{\sin \lambda(r-1)}{\lambda r^{2}}\right) \mathcal{F}_{0}\left(\left(-\Delta_{D}\right)^{\frac{1}{2}} f\right)(\lambda) d \lambda .
\end{aligned}
$$

This can in turn be written as

$$
\frac{2}{\pi} \int_{0}^{\infty} K_{1}^{T}(r, s)\left(\left(-\Delta_{D}\right)^{\frac{1}{2}} f\right)(s) s^{2} d s
$$

where $K_{1}^{T}$ is the transpose of the above kernel $K_{1}$,

$$
K_{1}^{T}(r, s)=\frac{1}{r s}\left(\frac{1}{r+s-2}-\frac{1}{r-s}\right)+\frac{1}{r^{2} s} \log \left|\frac{r-s}{r+s-2}\right| .
$$

Subtracting off $K_{0}^{T}(r, s)$ reduces matters to establishing bounds for the kernel

$$
K^{T}(r, s)=\frac{1}{r s}\left(\frac{1}{r+s-2}-\frac{1}{r+s}\right)+\frac{1}{r^{2} s} \log \left(\frac{r+s}{r+s-2}\right) .
$$

The first term is the same as above. The second term gives a bounded integral operator for $1<p<3$, since its transpose is bounded on $\frac{3}{2}<p<\infty$. 


\section{Proof of Theorem 1.1}

We begin by making the definition of the solution more precise. Let $I$ be a finite time interval containing 0 . As remarked above, (2.3) implies that radial $\dot{H}_{0}^{1}$ is closed under multiplication, so by $\dot{H}_{0}^{1}$ boundedness of $\exp \left(i t \Delta_{D}\right)$ we have

$$
\left\|\int_{0}^{t} e^{i(t-s) \Delta_{D}}|u|^{4} u(s) d s\right\|_{L_{t}^{\infty} \dot{H}_{0}^{1}(I \times \Omega)} \lesssim|I| \cdot\|u\|_{L_{t}^{\infty} \dot{H}_{0}^{1}(I \times \Omega)}^{5} .
$$

Therefore, if $u \in C\left(I ; \dot{H}_{0}^{1}(\Omega)\right)$, then the inhomogeneous term will also be in $\dot{H}_{0}^{1}(\Omega)$. This motivates the following

Definition 3.1 (Solution). Denote $F(u)=|u|^{4} u$. A radial function $u: I \times \Omega \rightarrow \mathbb{C}$ on a non-empty time interval $I \subset \mathbb{R}$ (possibly infinite or semi-infinite) is a strong $\dot{H}_{0}^{1}(\Omega)$ solution (or solution for short) to (1.1) if it lies in the class $C_{t}^{0} \dot{H}_{0}^{1}(I \times \Omega)$, and we have the Duhamel formula

$$
u\left(t_{1}\right)=e^{i\left(t_{1}-t_{0}\right) \Delta_{D}} u\left(t_{0}\right)-i \int_{t_{0}}^{t_{1}} e^{i\left(t_{1}-t\right) \Delta_{D}} F(u(t)) d t
$$

for all $t_{0}, t_{1} \in I$. We refer to the interval $I$ as the lifespan of $u$. We say that $u$ is a maximal-lifespan solution if the solution cannot be extended to any strictly larger interval. We say that $u$ is a global solution if $I=\mathbb{R}$.

Using (3.1) we can easily construct the local solution of (1.1) using a fixed point argument in $C_{t}^{0} \dot{H}_{0}^{1}(\Omega)$. Moreover, the lifespan of the local solution depends only on the $\dot{H}_{0}^{1}(\Omega)$ norm of the initial data. Existence of the global solution then follows quickly from the energy conservation property of the defocusing equation (1.1). Specifically, we have the following.

Theorem 3.1 (Global well-posedness). Let $u_{0} \in \dot{H}_{0}^{1}(\Omega)$ be spherically symmetric. Then there exists a unique global solution $u \in C\left(\mathbb{R} ; \dot{H}_{0}^{1}(\Omega)\right)$. Moreover, $\nabla u \in L_{t, l o c}^{q}$ $L_{x}^{r}(\mathbb{R} \times \Omega)$ for any admissible pair $(q, r)$, if $r<3$. For any $t \in \mathbb{R}$, we have

$$
E(u(t))=\frac{1}{2} \int_{\Omega}|\nabla u(t, x)|^{2} d x+\frac{1}{6} \int_{\Omega}|u(t, x)|^{6} d x=E\left(u_{0}\right) .
$$

For this global solution, scattering holds provided the global space-time $L^{10}$ norm is bounded. Precisely, suppose that $u$ satisfies

$$
\|u\|_{L_{t, x}^{10}([0, \infty) \times \Omega)}<\infty .
$$

Then $u$ scatters forward in time, i.e. there exists unique $v_{+} \in \dot{H}_{0}^{1}(\Omega)$ such that

$$
\lim _{t \rightarrow \infty}\left\|e^{i t \Delta_{D}} v_{+}-u(t)\right\|_{\dot{H}_{0}^{1}(\Omega)}=0 .
$$

The same statement holds backward in time.

The fact that global $L_{t, x}^{10}$ control of the norm implies finiteness of Strichartz norms and scattering is established by similar steps to those leading from (3.5) to (3.6) below. Furthermore, a standard continuity argument shows that if $\left\|u_{0}\right\|_{\dot{H}_{0}^{1}}<\epsilon$ for small $\epsilon$, then the corresponding solution scatters in both time directions. (see [7] for instance). 
Due to Theorem 3.1, the proof of Theorem 1.1 is reduced to showing that the $L_{t, x}^{10}$ norm of the solution over any compact time interval is bounded by a constant depending only on upper bounds for the initial energy. Theorem 1.1 is thus a consequence of the following.

Theorem 3.2. Assume $u \in \dot{H}_{0}^{1}(\Omega)$ is a spherically symmetric solution of (1.1) on a compact interval $\left[t_{-}, t_{+}\right]$. Suppose $E\left(u_{0}\right) \leq E$. Then

$$
\|u\|_{L_{t, x}^{10}\left(\left[t_{-}, t_{+}\right] \times \Omega\right)}<C(E) .
$$

The rest of this section will be devoted to the proof of Theorem 3.2. We begin with some useful conventions.

Convention. Let $0<\eta_{3} \ll \eta_{2} \ll \eta_{1} \ll \eta_{0} \ll 1$ be small constants to be determined. We use $c\left(\eta_{i}\right)$ to denote a small constant depending on $\eta_{i}$ such that $\eta_{i+1} \ll c\left(\eta_{i}\right) \ll \eta_{i}$. We use $C\left(\eta_{i}\right)$ to denote a large constant such that $\frac{1}{\eta_{i}} \ll C\left(\eta_{i}\right) \ll$ $\frac{1}{\eta_{i+1}}$. The constants $c\left(\eta_{i}\right)$ and $C\left(\eta_{i}\right)$ will sometimes vary from line to line, but the dependence is clear from the context. The notation $a \lesssim b$ will be used to mean that $a \leq C(E) b$, where $C(E)$ may depend on the energy upper-bound $E$.

We will use $\phi(x)$ to denote a radial smooth cutoff function such that

$$
\phi(x)= \begin{cases}1, & \text { if }|x| \leq 1 \\ 0, & \text { if }|x|>2\end{cases}
$$

We also denote $\phi_{<C}(x)=\phi\left(\frac{x}{C}\right), \phi_{>C}=1-\phi_{<C}$.

Since $\|u\|_{L_{t}^{\infty} L_{x}^{10}\left(\left[t_{-}, t_{+}\right] \times \Omega\right)} \lesssim E$, we decompose

$$
\left[t_{-}, t_{+}\right]=\bigcup_{j=1}^{J} I_{j}
$$

such that

$$
\eta_{0}<\|u\|_{L_{t, x}^{10}\left(I_{j} \times \Omega\right)} \leq 2 \eta_{0} .
$$

By Strichartz estimates on $I_{j}$, we have

$$
\begin{aligned}
\|\nabla u\|_{L_{t}^{8} L_{x}^{\frac{12}{5}}\left(I_{j} \times \Omega\right)} & \lesssim\left\|u\left(t_{i}\right)\right\|_{\dot{H}_{0}^{1}(\Omega)}+\left\|\nabla\left(|u|^{4} u\right)\right\|_{L_{t}^{\frac{40}{21}} L_{x}^{\frac{60}{49}}\left(I_{j} \times \Omega\right)} \\
& \lesssim 1+\|u\|_{L_{t, x}^{10}\left(I_{j} \times \Omega\right)}^{4}\|\nabla u\|_{L_{t}^{8} L_{x}^{\frac{12}{5}}\left(I_{j} \times \Omega\right)} \\
& \lesssim 1+\eta_{0}^{4}\|\nabla u\|_{L_{t}^{8} L_{x}^{\frac{12}{5}}\left(I_{j} \times \Omega\right)}
\end{aligned}
$$

Note we have used Proposition 2.2 to deduce the first inequality. By taking $\eta_{0}$ small we have

$$
\|\nabla u\|_{L_{t}^{8} L_{x}^{\frac{12}{5}}\left(I_{j} \times \Omega\right)} \lesssim 1
$$

A further application of Strichartz estimates yields that, for admissible pairs $(q, r)$ with $r<3$,

$$
\|\nabla u\|_{L_{t}^{q} L_{x}^{r}\left(I_{j} \times \Omega\right)} \lesssim 1
$$


Another fact implied by (3.4) and radial Sobolev embedding is that the length of all intervals here have uniform lower bound. Indeed, + since

$$
\begin{aligned}
\eta_{0}<\|u\|_{L_{t, x}^{10}\left(I_{j} \times \Omega\right)} & \leq\left|I_{j}\right|^{\frac{1}{10}}\|u\|_{L_{t}^{\infty} L_{x}^{10}\left(I_{j} \times \Omega\right)} \\
& \leq\left|I_{j}\right|^{\frac{1}{10}}\|u\|_{L_{t}^{\infty} \dot{H}_{0}^{1}\left(I_{j} \times \Omega\right)} \lesssim\left|I_{j}\right|^{\frac{1}{10}} .
\end{aligned}
$$

We have

$$
\left|I_{j}\right| \geq \eta_{1}, \forall j=1, \ldots, J
$$

Now let $u_{+}(t)=e^{i\left(t-t_{+}\right) \Delta_{D}} u\left(t_{+}\right), u_{-}(t)=e^{i\left(t-t_{-}\right) \Delta_{D}} u\left(t_{-}\right)$. We distinguish between two cases for each $I_{j}$ :

- $I_{j}$ is called exceptional if either

$$
\begin{aligned}
& \left\|u_{+}\right\|_{L_{t, x}^{10}\left(I_{j} \times \Omega\right)}>\eta_{0}^{10} \text { or } \\
& \left\|u_{-}\right\|_{L_{t, x}^{10}\left(I_{j} \times \Omega\right)}>\eta_{0}^{10} .
\end{aligned}
$$

- $I_{j}$ is called unexceptional if

$$
\left\|u_{ \pm}\right\|_{L_{t, x}^{10}\left(I_{j} \times \Omega\right)} \leq \eta_{0}^{10} .
$$

Since $\left\|u_{ \pm}\right\|_{\left.L_{t, x}^{10}\left(\left[t_{-}, t_{+}\right]\right) \times \Omega\right)} \lesssim\left\|\nabla u_{ \pm}\right\|_{\left.L_{t}^{10} L_{x}^{\frac{30}{13}}\left(\left[t_{-}, t_{+}\right]\right) \times \Omega\right)} \lesssim\left\|u\left(t_{ \pm}\right)\right\|_{\dot{H}_{0}^{1}}$, the number of exceptional intervals is bounded by $C\left(\eta_{0}, E\right)$. We thus need to control only the number of unexceptional intervals.

We now focus on the mass concentration property about unexceptional intervals. We begin with local mass conservation.

Let $\phi$ be the smooth cutoff function defined in (3.3), and $\phi_{R}(x)=\phi(x / R)$. Define the local mass of $u$ to be

$$
M_{R}(t)=\int_{|x| \geq 1}|u(t, x)|^{2} \phi_{R}^{2}(x) d x .
$$

Then we have

Lemma 3.1 (Local mass conservation).

$$
\left|\frac{d}{d t} M_{R}^{\frac{1}{2}}(t)\right| \lesssim \frac{1}{R}
$$

Proof. Using the equation in (1.1), we compute

$$
\begin{aligned}
\frac{d}{d t} M_{R}(t) & =2 \operatorname{Re} \int_{|x| \geq 1} u_{t} \bar{u} \phi_{R}^{2}(x) d x \\
& =-2 \operatorname{Im} \int_{|x| \geq 1} \Delta u \cdot \bar{u} \phi_{R}^{2}(x) d x \\
& =\frac{2}{R} \operatorname{Im} \int_{|x| \geq 1} \nabla u \bar{u} \phi_{R}(x)(\nabla \phi)_{R}(x) d x .
\end{aligned}
$$


Therefore

$$
\begin{aligned}
\left|\frac{d}{d t} M_{R}(t)\right| & \lesssim \frac{2}{R}\|\nabla u\|_{L^{2}(|x| \geq 1)}\left\|u \phi_{R}\right\|_{L^{2}(|x| \geq 1)} \\
& \lesssim \frac{1}{R} M_{R}^{\frac{1}{2}}(t) .
\end{aligned}
$$

From here, (3.7) follows directly.

We next establish the important

Lemma 3.2 (Mass concentration on unexceptional intervals). Let $I$ be an unexceptional interval. Then for any $t \in I$,

$$
\int_{|x|<\frac{1}{\eta_{3}}|I|^{\frac{1}{2}}}|u(t, x)|^{2} d x \geq c\left(\eta_{2}\right)|I|
$$

Proof. Denote

$$
I=[a, b] .
$$

Without loss of generality, we assume ${ }^{3}$

$$
\|u\|_{L_{t, x}^{10}\left(\left[\frac{a+b}{2}, b\right] \times \Omega\right)} \geq \frac{\eta_{0}}{2} .
$$

By the Duhamel formula

$$
u(t)=u_{-}(t)-i \int_{t_{-}}^{a} e^{i(t-s) \Delta_{D}} F(u)(s) d s-i \int_{a}^{t} e^{i(t-s) \Delta_{D}} F(u)(s) d s .
$$

We define

$$
\begin{aligned}
w(t) & :=i \int_{t_{-}}^{a} e^{i(t-s) \Delta_{D}} F(u)(s) d s \\
& =-u(t)+u_{-}(t)-i \int_{a}^{t} e^{i(t-s) \Delta_{D}} F(u)(s) d s .
\end{aligned}
$$

We next observe that $w$ has certain bounds. By Strichartz estimates and the steps leading from (3.5) to (3.6),

$$
\sup _{t}\|w(t, \cdot)\|_{\dot{H}_{0}^{1}(\Omega)} \lesssim 1, \quad \forall t \in I .
$$

Moreover, we have

$$
\begin{gathered}
\left\|u_{-}\right\|_{L_{t, x}^{10}\left(\left[\frac{a+b}{2}, b\right] \times \Omega\right)} \\
\left\|\int_{a}^{t} e^{i(t-s) \Delta_{D}} F(u)(s) d s\right\|_{L_{t, x}^{10}\left(\left[\frac{a+b}{2}, b\right] \times \Omega\right)} \lesssim \eta_{0}^{4} \leq \eta_{0}^{2} .
\end{gathered}
$$

By the triangle inequality we then have

$$
\|w\|_{L_{t, x}^{10}\left(\left[\frac{a+b}{2}, b\right] \times \Omega\right)} \geq \frac{\eta_{0}}{4} .
$$

\footnotetext{
${ }^{3}$ Otherwise if this holds for $\left[a, \frac{a+b}{2}\right]$, and we apply a similar argument by just reversing the time direction.
} 
We now show the main $L_{t, x}^{10}$-norm is localized in low frequencies. To this end, we use dispersive estimates to control the high frequencies as follows:

$$
\begin{aligned}
\left\|P_{>C\left(\eta_{2}\right)|I|^{-\frac{1}{2}}} w\right\|_{L_{t, x}^{10}\left(\left[\frac{a+b}{2}, b\right] \times \Omega\right)} & \|\| \int_{t_{-}}^{a} e^{i(t-s) \Delta_{D}} P_{>C\left(\eta_{2}\right)|I|^{-\frac{1}{2}}} F(u(s, \cdot)) d s \|_{L_{t, x}^{10}\left(\left[\frac{a+b}{2}, b\right] \times \Omega\right)} \\
& \lesssim|I|^{\frac{1}{10}} \int_{t_{-}}^{a}\left|\frac{a+b}{2}-s\right|^{-\frac{6}{5}}\left\|P_{>C\left(\eta_{2}\right)|I|^{-\frac{1}{2}}} F(u(s, \cdot))\right\|_{L_{x}^{\frac{10}{9}}(\Omega)} d s \\
& \lesssim|I|^{-\frac{1}{10}}\|F(u)\|_{L_{t}^{\infty} L_{x}^{\frac{10}{9}}\left(\left[t_{-}, t_{+}\right] \times \Omega\right)} .
\end{aligned}
$$

First consider the case that $\eta_{2}|I|^{\frac{1}{2}} \geq 2$. We estimate the norm of $F(u)$ in different spatial regimes. First,

$$
\begin{aligned}
\left\|P_{>C\left(\eta_{2}\right)|I|^{-\frac{1}{2}}} \phi_{<\eta_{2}|I|^{\frac{1}{2}}} F(u)\right\|_{L_{x}^{\frac{10}{9}}(\Omega)} \\
\quad \lesssim\left\|\phi_{<\eta_{2}|I|^{\frac{1}{2}}} F(u)\right\|_{L_{x}^{\frac{10}{9}}(\Omega)} \\
\quad \lesssim\|F(u)\|_{L_{x}^{\frac{6}{5}}(\Omega)}\left\|\phi_{<\eta_{2}|I|^{\frac{1}{2}}}\right\|_{L_{x}^{15}(\Omega)} \\
\quad \lesssim \eta_{2}^{\frac{1}{5}}|I|^{\frac{1}{10}}\|u\|_{L_{x}^{6}(\Omega)}^{5} \\
\quad \lesssim \eta_{2}^{\frac{1}{5}}|I|^{\frac{1}{10}}
\end{aligned}
$$

Next, using the radial Sobolev embedding

$$
\left\||x|^{\frac{1}{5}} u\right\|_{L_{x}^{10}} \lesssim\|\nabla u\|_{L_{x}^{2}}
$$

together with the Bernstein inequality and Proposition 2.2, we estimate

$$
\begin{aligned}
& \left\|P_{>C\left(\eta_{2}\right)|I|^{-\frac{1}{2}}}\left(\phi_{>\eta_{2}|I|^{\frac{1}{2}}} F(u)\right)\right\|_{L_{x}^{\frac{10}{9}}(\Omega)} \\
& \lesssim c\left(\eta_{2}\right)|I|^{\frac{1}{2}}\left\|\nabla\left(\phi_{>\eta_{2}|I|^{\frac{1}{2}}} F(u)\right)\right\|_{L_{x}^{\frac{10}{9}}(\Omega)} \\
& \lesssim c\left(\eta_{2}\right)|I|^{\frac{1}{2}}\left(\left\|\nabla \phi_{>\eta_{2}|I|^{\frac{1}{2}}}\right\|_{L_{x}^{15}(\Omega)}\|u\|_{L_{x}^{6}(\Omega)}^{5}+\left\|\phi_{>\eta_{2}|I|^{\frac{1}{2}}} u\right\|_{L_{x}^{10}(\Omega)}^{4}\|\nabla u\|_{L_{x}^{2}(\Omega)}\right) \\
& \lesssim c\left(\eta_{2}\right)|I|^{\frac{1}{2}}\left(\eta_{2}|I|^{\frac{1}{2}}\right)^{-\frac{4}{5}}\left(\|u\|_{L_{x}^{6}(\Omega)}+\|\nabla u\|_{L_{x}^{2}(\Omega)}\right)^{5} \\
& \lesssim c\left(\eta_{2}\right) \eta_{2}^{-\frac{4}{5}}|I|^{\frac{1}{10}} \leq \eta_{2}^{2}|I|^{\frac{1}{10}} \text {. }
\end{aligned}
$$

Therefore

$$
\left\|P_{>C\left(\eta_{2}\right)|I|^{-\frac{1}{2}}} w\right\|_{L_{t, x}^{10}\left(\left[\frac{a+b}{2}, b\right] \times \Omega\right)} \leq \frac{\eta_{0}}{100} .
$$

In case $\eta_{2}|I|^{\frac{1}{2}} \leq 2$, applying a similar argument without the cutoff $\phi$ yields the better bound $c\left(\eta_{2}\right)|I|^{\frac{1}{2}}$.

This combines with (3.9) give

$$
\left\|P_{<C\left(\eta_{2}\right)|I|^{-\frac{1}{2}}} w\right\|_{L_{t, x}^{10}\left(\left[\frac{a+b}{2}, b\right] \times \Omega\right)} \geq \frac{\eta_{0}}{8} .
$$

Recalling the definition of $w$, the boundedness of $P,(3.8)$ and (3.9), and the condition for unexceptional intervals, we have

$$
\left\|P_{<C\left(\eta_{2}\right)|I|^{-\frac{1}{2}}} u\right\|_{L_{t, x}^{10}\left(\left[\frac{a+b}{2}, b\right] \times \Omega\right)} \geq \frac{\eta_{0}}{10} .
$$


On the other hand, interpolation and the lower bound for $|I|$ yield

$$
\begin{aligned}
\| \phi & >\frac{1}{\eta_{3}}|I|^{\frac{1}{2}} u \|_{L_{t, x}^{10}\left(\left[\frac{a+b}{2}, b\right] \times \Omega\right)} \\
& \lesssim|I|^{\frac{1}{10}}\left\|\phi_{>\frac{1}{\eta_{3}}|I|^{\frac{1}{2}}} u\right\|_{L_{t}^{\infty} L_{x}^{10}\left(\left[\frac{a+b}{2}, b\right] \times \Omega\right)} \\
& \lesssim|I|^{\frac{1}{10}}\left\|\phi_{>\frac{1}{\eta_{3}}|I|^{\frac{1}{2}}} u\right\|_{L_{t}^{\frac{3}{5}} L_{x}^{6}\left(\left[\frac{a+b}{2}, b\right] \times \Omega\right)}\left\|\phi_{>\frac{1}{\eta_{3}}|I|^{\frac{1}{2}}} u\right\|_{L_{t}^{\infty} L_{x}^{\infty}\left(\left[\frac{a+b}{2}, b\right] \times \Omega\right)}^{\frac{2}{5}} \\
& \lesssim|I|^{\frac{1}{10}}\left(\frac{1}{\eta_{3}}|I|^{\frac{1}{2}}\right)^{-\frac{1}{5}} \\
& \leq \eta_{0}^{2} .
\end{aligned}
$$

Thus, (3.12) can be improved to

$$
\left\|P_{<C\left(\eta_{2}\right)|I|^{-\frac{1}{2}}} \phi_{<\frac{1}{\eta_{3}}|I|^{\frac{1}{2}}} u\right\|_{L_{t, x}^{10}\left(\left[\frac{a+b}{2}, b\right] \times \Omega\right)} \geq \frac{\eta_{0}}{20} .
$$

From this, the mass concentration follows quickly. Indeed, using the Bernstein and Hölder inequalities yields

$$
\begin{aligned}
\frac{\eta_{0}}{20} & \leq\left\|P_{<C\left(\eta_{2}\right)|I|^{-\frac{1}{2}}} \phi_{<\frac{1}{\eta_{3}}|I|^{\frac{1}{2}}} u\right\|_{L_{t, x}^{10}\left(\left[\frac{a+b}{2}, b\right] \times \Omega\right)} \\
& \lesssim|I|^{\frac{1}{10}}\left(C\left(\eta_{2}\right)|I|^{-\frac{1}{2}}\right)^{3\left(\frac{1}{2}-\frac{1}{10}\right)}\left\|\phi_{<\frac{1}{\eta_{3}}|I|^{\frac{1}{2}}} u\right\|_{L_{t}^{\infty} L_{x}^{2}\left(\left[\frac{a+b}{2}, b\right] \times \Omega\right)} \\
& \lesssim C\left(\eta_{2}\right)|I|^{-\frac{1}{2}}\left\|\phi_{<\frac{1}{\eta_{3}}|I|^{\frac{1}{2}}} u\right\|_{L_{t}^{\infty} L_{x}^{2}\left(\left[\frac{a+b}{2}, b\right] \times \Omega\right)} .
\end{aligned}
$$

Thus, there exists $t_{0} \in I$ such that

$$
\left\|u\left(t_{0}\right)\right\|_{L^{2}\left(1 \leq|x|<\frac{1}{\eta_{3}}|I|^{\frac{1}{2}}\right)}>c\left(\eta_{2}\right)|I|^{\frac{1}{2}} .
$$

Using (3.7) we obtain

$$
\|u(t)\|_{L^{2}\left(1 \leq|x|<\frac{1}{\eta_{3}}|I|^{\frac{1}{2}}\right)}>c\left(\eta_{2}\right)|I|^{\frac{1}{2}}, \quad \forall t \in I .
$$

Proposition 3.1 (Morawetz inequality). Let I be a time interval, and let $A \geq 1$. Then

$$
\int_{I} \int_{1 \leq|x| \leq A|I|^{\frac{1}{2}}} \frac{|u(t, x)|^{6}}{|x|} d x d t \lesssim A|I|^{\frac{1}{2}} .
$$

Proof. We begin with the local momentum conservation identity

$$
\partial_{t} \operatorname{Im}\left(\partial_{k} u \bar{u}\right)=-2 \partial_{j} R e\left(\partial_{k} u \partial_{j} \bar{u}\right)+\frac{1}{2} \partial_{k} \Delta\left(|u|^{2}\right)-\frac{2}{3} \partial_{k}|u|^{6} .
$$

Let $a(x)=|x| \phi_{<R}(x)$, so that $a$ is a radial function. Let $a_{j k}=\partial_{j} \partial_{k} a$. Observe that for $1 \leq|x| \leq R$,

$$
a_{j k}(x) \text { is positive definite, } \quad \nabla a(x)=\frac{x}{|x|}, \quad \Delta^{2} a(x)<0 .
$$

In the region $|x| \geq R, a(x)$ has the rough bound

$$
\left|\partial_{k} a\right| \lesssim 1, \quad\left|a_{j k}\right| \lesssim \frac{1}{R}, \quad\left|\Delta^{2} a\right| \lesssim \frac{1}{R^{3}}
$$


We multiply the first term in (3.13) by $\partial_{k} a$ and integrate over $\Omega$ to obtain

$$
\begin{aligned}
& -2 \int_{|x| \geq 1} \partial_{j} \operatorname{Re}\left(\partial_{k} u \partial_{j} \bar{u}\right) \partial_{k} a d x \\
& =2 \int_{|x| \geq 1} \operatorname{Re}\left(\partial_{k} u \partial_{j} \bar{u}\right) a_{j k} d x+2 \int_{|x|=1} \operatorname{Re}\left(\partial_{k} u \partial_{j} \bar{u}\right) x_{j} x_{k} d \sigma \\
& =2 \int_{1 \leq|x| \leq R} \operatorname{Re}\left(\partial_{k} u \partial_{j} \bar{u}\right) a_{j k} d x+2 \int_{|x| \geq R} \operatorname{Re}\left(\partial_{k} u \partial_{j} \bar{u}\right) a_{j k} d x \\
& \quad+2 \int_{|x|=1}|\nabla u|^{2} d \sigma(x) \\
& \geq-\frac{1}{R}\|\nabla u\|_{L^{2}(\Omega)}^{2}+2 \int_{|x|=1}|\nabla u|^{2} d \sigma(x) .
\end{aligned}
$$

We do the same for the second term in (3.13), and use the Dirichlet condition to calculate

$$
\begin{aligned}
& \frac{1}{2} \int_{|x| \geq 1} \partial_{k} \Delta\left(|u|^{2}\right) \partial_{k} a d x \\
& \quad=-\frac{1}{2} \int_{|x| \geq 1} \Delta\left(|u|^{2}\right) \Delta a d x-\frac{1}{2} \int_{|x|=1} \Delta\left(|u|^{2}\right) d \sigma \\
& \quad=-\frac{1}{2} \int_{|x| \geq 1}|u|^{2} \Delta^{2} a d x-\int_{|x|=1}|\nabla u|^{2} d \sigma(x) \\
& \quad=-\frac{1}{2} \int_{1 \leq|x| \leq R}|u|^{2} \Delta^{2} a d x-\frac{1}{2} \int_{|x| \geq R}|u|^{2} \Delta^{2} a d x-\int_{|x|=1}|\nabla u|^{2} d \sigma(x) \\
& \quad \geq-\frac{C}{R}\|u\|_{6}^{2}-\int_{|x|=1}|\nabla u|^{2} d \sigma(x) .
\end{aligned}
$$

Similarly for the third term in (3.13),

$$
\begin{aligned}
-\frac{2}{3} \int_{|x| \geq 1} \partial_{k}\left(|u|^{6}\right) \partial_{k} a d x & =\frac{2}{3} \int_{|x| \geq 1}|u|^{6} \Delta a d x \\
& =\frac{4}{3} \int_{1 \leq|x| \leq R} \frac{|u|^{6}}{|x|} d x+\frac{2}{3} \int_{|x| \geq R}|u|^{6} \Delta a d x \\
& \geq \frac{4}{3} \int_{1 \leq|x| \leq R} \frac{|u|^{6}}{|x|} d x-\frac{1}{R}\|u\|_{6}^{6} .
\end{aligned}
$$

Notice also that

$$
\left|\int_{|x| \geq 1} \operatorname{Im}\left(\partial_{k} u \bar{u}\right) \partial_{k} a d x\right| \lesssim\left\|\partial_{k} u\right\|_{L^{2}(\Omega)}\|u\|_{L^{6}(\Omega)}\left\|\partial_{k} a\right\|_{L^{3}(\Omega)} \lesssim R .
$$

Integrating (3.13) over $I \times \Omega$ we obtain

$$
\int_{I} \int_{1 \leq|x| \leq R} \frac{|u|^{6}}{|x|} d x d t \lesssim \frac{|I|}{R}+R
$$


Taking $R=A|I|^{\frac{1}{2}}$, since $A \geq 1$ we have

$$
\int_{I} \int_{1 \leq|x| \leq A|I|^{\frac{1}{2}}} \frac{|u|^{6}}{|x|} d x d t \lesssim A|I|^{\frac{1}{2}}
$$

Lemma 3.3. Let $J$ be an interval that contains a contiguous collection $\bigcup_{j} I_{j}$ of unexceptional intervals. Then we have

$$
\sum\left|I_{j}\right|^{\frac{1}{2}} \leq C\left(\eta_{2}, \eta_{3}\right)|J|^{\frac{1}{2}}
$$

Proof. We apply mass concentration on each of the time intervals $I_{j}$ to get

$$
c\left(\eta_{2}\right)\left|I_{j}\right| \leq \int_{1 \leq|x| \leq \frac{1}{\eta_{3}}\left|I_{j}\right|^{\frac{1}{2}}}|u(t, x)|^{2} d x \lesssim\left(\int_{1 \leq|x| \leq \frac{1}{\eta_{3}}\left|I_{j}\right|^{\frac{1}{2}}} \frac{|u(t, x)|^{6}}{|x|} d x\right)^{\frac{1}{3}}\left(\frac{1}{\eta_{3}}\left|I_{j}\right|^{\frac{1}{2}}\right)^{\frac{7}{3}} .
$$

Therefore

$$
c\left(\eta_{2}, \eta_{3}\right)\left|I_{j}\right|^{-\frac{1}{2}} \lesssim \int_{1 \leq|x| \leq \frac{1}{\eta_{3}}\left|I_{j}\right|^{\frac{1}{2}}} \frac{|u(t, x)|^{6}}{|x|} d x .
$$

We integrate in time over $I_{j}$ and sum over $j$. The Morawetz inequality then gives

$$
c\left(\eta_{2}, \eta_{3}\right) \sum\left|I_{j}\right|^{\frac{1}{2}} \lesssim \frac{1}{\eta_{3}}|J|^{\frac{1}{2}}
$$

which implies (3.14).

From the estimate (3.14), one easily sees that the number of unexceptional intervals will be under control if the size of the intervals decrease slowly, for example, they remain constant or decrease polynomially. The only scenario that is consistent with estimate (3.14) is exponential decrease. Indeed, applying the algorithm argument introduced by Bourgain [6], one can extract a sequence of exponentially decaying intervals converging on a point. ${ }^{4}$ See also [19] for a simplified presentation for this part of argument. Here, we will just record the result and omit the proof.

Proposition 3.2. Let $u$ be the solution under consideration. Then there exists a time $t_{*} \in\left[t_{-}, t_{+}\right]$and distinct unexceptional intervals $I_{j 1}, \ldots, I_{j K}$ for some $K>$ $c\left(\eta_{2}, \eta_{3}\right) \log J$ such that

$$
\left|I_{j 1}\right| \geq 2\left|I_{j 2}\right| \geq 4\left|I_{j 3}\right| \geq \cdots \geq 2^{K-1}\left|I_{j K}\right|
$$

and such that $\operatorname{dist}\left(t_{*}, I_{j k}\right) \leq C\left(\eta_{2}, \eta_{3}\right)\left|I_{j k}\right|$ for all $1 \leq k \leq K$.

At this point, we can get the control on $K$ as well as $J$. The detailed argument can be found in [6] or [19]. We will give a brief sketch for the sake of completeness.

From mass concentration on $I_{j k}$, Lemma 3.2, and local mass conservation (by suitably adjusting the constant), we see that at this fixed $t_{*}$,

$$
\int_{1 \leq|x| \leq C\left(\eta_{2}, \eta_{3}\right)\left|I_{j k}\right|^{\frac{1}{2}}}\left|u\left(t_{*}, x\right)\right|^{2} d x>c\left(\eta_{2}\right)\left|I_{j k}\right| .
$$

\footnotetext{
${ }^{4}$ In our case, this is a precise description since we have a uniform lower bound on the length of the intervals $I_{j}$.
} 
On the other hand, using the following upper bound

$$
\int_{1 \leq|x| \leq C\left(\eta_{2}, \eta_{3}\right)\left|I_{j k}\right|^{\frac{1}{2}}}\left|u\left(t_{*}, x\right)\right|^{2} d x \leq C\left(\eta_{2}, \eta_{3}\right)\left|I_{j k}\right|,
$$

and dyadic shrinking property of $\left|I_{j k}\right|$, we know that for each $k \in[1, K]$ there exist at most $N=\log C\left(\eta_{2}, \eta_{3}\right)$ annuli such that the main mass in (3.16) occupies only these finite annuli:

$$
\int_{\left|I_{j(k+N)}\right|^{\frac{1}{2}} \leq \frac{|x|}{C\left(\eta_{2}, \eta_{3}\right)} \leq\left|I_{j k}\right|^{\frac{1}{2}}}\left|u\left(t_{*}, x\right)\right|^{2} d x>c\left(\eta_{2}\right)\left|I_{j k}\right| .
$$

By Hölder's inequality, the $L_{x}^{6}$ norm also concentrates on this collection of annuli with constant lower bound. Summing over $k$ produces at most $N \int\left|u\left(t_{*}, x\right)\right|^{6}$, which yields the bound on $K$. We thus have

Theorem 3.3. There exists $C\left(E, \eta_{0}, \eta_{1}, \eta_{2}, \eta_{3}\right)$ such that

$$
\#\left\{I_{j}, I_{j} \text { is unexceptional }\right\} \leq C\left(E, \eta_{0}, \eta_{1}, \eta_{2}, \eta_{3}\right) \text {. }
$$

This, combined with the fact that the number of exceptional intervals is finite, proves Theorem 1.1 .

\section{The energy supercritical problem}

For spherically symmetric function $f \in \dot{H}_{0}^{1}(\Omega)$, the bound

$$
\|f\|_{L_{x}^{\infty}(\Omega)} \leq\left\||x|^{\frac{1}{2}} f\right\|_{L_{x}^{\infty}(\Omega)} \lesssim\|f\|_{\dot{H}_{0}^{1}(\Omega)},
$$

means that any supercritical nonlinearity $|u|^{p} u$ for any $p>4$ can still be viewed as "critical". As a consequence, the proof of Theorem 1.1 can be applied to the supercritical case after some minor modifications. More specifically, we consider the following energy supercritical NLS in $\Omega$ with the Dirichlet boundary condition:

$$
\left\{\begin{array}{l}
i \partial_{t} u+\Delta u=|u|^{p} u, p>4 \\
\left.u(t, x)\right|_{\mathbb{R} \times \partial \Omega}=0 \\
u(0, x)=u_{0}(x)
\end{array}\right.
$$

We then have the following result.

Theorem 4.1. Let $u_{0} \in \dot{H}_{0}^{1}(\Omega)$ be spherically symmetric. Then there exists a unique solution $u \in C\left(\mathbb{R} ; \dot{H}_{0}^{1}\right)$ to $(4.1)$, and for this solution it holds that

Energy Conservation:

$$
E(u(t))=\frac{1}{2}\|\nabla u(t)\|_{L_{x}^{2}(\Omega)}^{2}+\frac{1}{p+2}\|u(t)\|_{L_{x}^{p+2}(\Omega)}^{p+2}=E\left(u_{0}\right),
$$

Global space-time Bound:

$$
\|u\|_{L_{t, x}^{10}(\mathbb{R} \times \Omega)} \leq C\left(\left\|u_{0}\right\|_{\dot{H}_{0}^{1}(\Omega)}\right) .
$$

Moreover, there exist unique $v_{ \pm} \in \dot{H}_{0}^{1}(\Omega)$ such that

$$
\lim _{t \rightarrow \pm \infty}\left\|u(t)-e^{i t \Delta_{D}} v_{ \pm}\right\|_{\dot{H}_{0}^{1}(\Omega)}=0 .
$$




\section{The case with inhomogeneous $H_{0}^{1}(\Omega)$ data}

In this section, we point out that for the energy critical problem, Bourgain's argument is not needed if we assume inhomogeneous data $u_{0} \in H_{0}^{1}(\Omega)$.

Indeed, consider (1.1) with radial $u_{0} \in H_{0}^{1}(\Omega)$. By taking $a(x)=|x|$ and using almost the same computation as in Proposition 3.1, we arrive at

$$
\left\||x|^{-\frac{1}{6}} u\right\|_{L_{t, x}^{6}(\mathbb{R} \times \Omega)}^{6} \lesssim\left\|u_{0}\right\|_{H_{0}^{1}(\Omega)}^{2} .
$$

This interpolates with the radial Sobolev embedding (2.3) to immediately yield

$$
\|u\|_{L_{t, x}^{10}(\mathbb{R} \times \Omega)} \lesssim_{u} 1
$$

which is enough to prove scattering.

Finally, the same argument with small changes in numerology applies to the energy supercritical case (4.1). We omit the details.

\section{Acknowledgments}

Dong Li was supported by a start up fund in UBC. Hart Smith is supported by NSF Grant no. DMS-0654415. Xiaoyi Zhang was supported by an Alfred P. Sloan Research Fellowship. We all thank the anonymous referee for the very helpful comments about the draft of the paper.

\section{References}

[1] R. Anton, Global existence for defocusing cubic NLS and Gross-Pitaveskii equations in exterior domains, J. Math. Pures Appl. 89(4) (2008), 335-354.

[2] N. Burq, P. Gérad and N. Tzvetkov, On nonlinear Schrödinger equations in exterior domains, Ann. Inst. H. Poincaré Anal. Non Linéaire 21(3) (2004), 295-318.

[3] N. Burq, G. Lebeau and F. Planchon, Global existence for energy critical waves in 3-D domains, J. Amer. Math. Soc. 21(3) (2008), 831-845.

[4] M.D. Blair, H.F. Smith and C.D. Sogge, Strichartz estimates and the nonlinear Schrödinger equation on manifolds with boundary, to appear in Math. Ann.

[5] M.D. Blair, H.F. Smith and C.D. Sogge, Strichartz estimates for the wave equation on manifolds with boundary, Ann. Inst. H. Poincaré Anal. Non Linéaire 26(5) (2009), 1817-1829.

[6] J. Bourgain, Global well-posedness of defocusing $3 D$ critical NLS in the radial case, J. Amer. Math. Soc. 12 (1999), 145-171.

[7] T. Cazenave and F. B. Weissler, The Cauchy problem for the critical nonlinear Schrödinger equation in $H^{s}$, Nonlinear Anal. T. M. A. 14 (1990), 807-836.

[8] J. Colliander, M. Keel, G. Staffilani, H. Takaoka and T. Tao, Global well-posedness and scattering for the energy-critical nonlinear Schrödinger equation in $\mathbb{R}^{3}$, Ann. Math. 166 (2007), $1-100$.

[9] P. Constantin and J.C. Saut, Local smoothing properties of dispersive equations, J. Amer. Math. Soc. 1 (1988), 413-439.

[10] O. Ivanovici, Precise smoothing effect in the exterior of balls, Asymptot. Anal. 53(4) (2007), 189-208.

[11] O. Ivanovici, On the Schrödinger equation outside strictly convex obstacles, Anal. PDE 3 (2010), 261-293.

[12] M. Keel and T. Tao, Endpoint Strichartz Estimates, Amer. Math. J. 120 (1998), 955-980.

[13] C.E. Kenig and F. Merle, Global well-posedness, scattering, and blowup for the energy-critical, focusing, non-linear Schrödinger equation in the radial case, Invent. Math. 166 (2006), 645-675.

[14] R. Killip and M. Visan, The focusing energy-critical nonlinear Schrödinger equation in dimensions five and higher, Amer. J. Math. 132 (2010), 361-424. 
[15] E. Ryckman and M. Visan, Global well-posedness and scattering for the defocusing energycritical nonlinear Schrödinger equation in $\mathbb{R}^{1+4}$, Amer. J. Math. 129 (2007), 1-60.

[16] P. Sjölin, Regularity of solutions to Schrödinger equations, Duke Math J. 55 (1987), 699-715.

[17] H.F. Smith and C.D. Sogge, On the critical semilinear wave equation outside convex obstacles, J. Amer. Math. Soc. 8 (1995), 879-916.

[18] H.F. Smith and C.D. Sogge, On the $L^{p}$ norm of spectral clusters for compact manifolds with boundary, Acta Math. 198 (2007), 107-153.

[19] T. Tao, Global well-posedness and scattering for the higher-dimensional energy-critical nonlinear Schrödinger equation for radial data, New York J. Math. 11 (2005), 57-80.

[20] L. Vega, Schrödinger equations: pointwise convergence to the initial data. Proc. Amer. Math. Soc 102 (1988), 874-878.

[21] M. Visan, The defocusing energy-critical nonlinear Schrödinger equation in higher dimensions, Duke Math. J. 138(2) (2007), 281-374.

Department of Mathematics, University of British Columbia, Vancouver, BC, Canada V6T 1 Z2

E-mail address: mpdongli@gmail.com

Department of Mathematics, University of Washington, Seattle, WA 98195-4350

E-mail address: hart@math.washington.edu

Department of Mathematics, University of Iowa, 14 Maclean Hall, Iowa City, USA 52242 and Chinese Academy of Science, Beijing, People's Republic of China

E-mail address: zh.xiaoyi@gmail.com 